Surveillance post-radiofrequency ablation for small renal masses

\title{
Surveillance post-radiofrequency ablation for small renal masses: Recurrence and followup
}

Cameron J. Lam ${ }^{1}$; Nathan C. Wong ${ }^{1}$; MauriceVoss²; Oleg Mironov²; Michael Connolly²; Edward Matsumoto ${ }^{1}$; Anil Kapoor ${ }^{1}$

${ }^{1}$ Department of Surgery, Division of Urology, McMaster University, Hamilton, ON, Canada; ${ }^{2}$ Department of Radiology, McMaster University, Hamilton, ON, Canada

Cite as: Can Urol Assoc J 2020 June 16; Epub ahead of print. http://dx.doi.org/10.5489/cuaj.6374

Published online June 16, 2020

$* * *$

\section{Abstract}

Introduction: Small renal masses (SRMs), enhancing tumors $<4 \mathrm{~cm}$ in diameter, are suspicious for renal cell carcinoma (RCC). The incidence of SRMs have risen with the increased quality and frequency of imaging. Partial nephrectomy is widely accepted as a nephron-sparing approach for the management of clinically localized RCC, with a greater than $90 \%$ disease-specific survival for stage T1a. Radiofrequency ablation (RFA) has been emerging as an alternative management strategy, with evidence suggesting RFA as a safe alternative for SRMs. We aimed to evaluate the time to recurrence and recurrence rates of SRMs treated with RFA at our institution.

Methods: A retrospective review between October 2011 and May 2019 identified 141 patients with a single SRM treated with RFA at Hamilton Health Sciences and St. Joseph's Healthcare Hamilton. Patients with familial syndromes and distant metastases were excluded. Repeat RFAs of the ipsilateral kidney for incomplete ablation were not considered a new procedure. The primary variable measured was time from initial ablation to recurrence. A Cox proportional hazard regression model was used to identify possible prognostic variables for tumor recurrence defined a priori, including age, gender, mass size, RENAL nephrometry, and PADUA scores. Results: The overall average age of our patients was $69.0 \pm 11.1$ years, with $71.6 \%$ being male. Average tumor size was $2.6 \pm 0.8 \mathrm{~cm}$. There was a total of $22 / 154$ total recurrences $(15.6 \%)$ postRFA. Median followup time was 67 (18-161) months. Those with new recurrences had median time to recurrence of 15 months and no recurrence beyond 53 months. Thirteen of 141 patients had residual disease $(9.2 \%)$ and were identified within the first eight months post-RFA. The only prognostic variable identified as a predictor of residual disease was tumor size (hazard ratio $2.265 ; \mathrm{p}<0.001)$. 
Conclusions: This study shows the risk of a new recurrence following RFA for SRMs is $6.4 \%$. Most recurrences $(9.2 \%)$ were a result of residual tumor at the ablation site identified within the first eight months post-RFA. No recurrences were identified beyond 53 months, with a total median followup time of 67 months. Tumor size alone, without need for complex scoring systems, may serve as a predictor of incomplete ablation following RFA and could be used to assist in shared decision-making on management strategies.

\section{Introduction}

Small renal masses (SRMs) are defined clinically as solid enhancing tumours $\leq 4 \mathrm{~cm}$ consistent to stage T1a N0 M0 renal cell carcinoma (RCC). ${ }^{1-3}$ The incidence of SRMs has increased in part due to the increased use of cross-sectional imaging; however, mortality rates have not followed similar patterns. ${ }^{4-6}$ Currently, the standard treatment therapy for local RCC has been partial nephrectomy and radical nephrectomy depending on the location and characteristics of the lesion. ${ }^{2,3,6-8}$ Treatment for these SRMs has changed tremendously over the past decades with radiofrequency ablation (RFA) emerging as a treatment option for soft tissue tumours in the 1990s. ${ }^{9}$ Recently RFA and active surveillance (AS) have been used as alternative management strategies dependant on patient preference and surgical risk factors. ${ }^{2,8,10-13}$ Outcomes from surgical management with partial nephrectomy are excellent for T1a RCC with over 90\% disease-specific survival. ${ }^{7,14}$ Long-term follow-up and outcomes have not been as well studied for RFA therapy for SRMs though there have been a few reported studies suggesting that RFA is a safe and effective treatment option for SRMs less than $3 \mathrm{~cm}$ in diameter. ${ }^{12,15}$

SRMs found in the elderly, frail, or patients with a solitary kidney may not be good candidates for surgical management and may be better managed under active surveillance (AS). Those patients unwilling to accept the inherent risks of AS or are poor surgical candidates may benefit from RFA. Risk of surgery should be weighed against life expectancy, malignant potential, and chance of recurrence. Approximately 20-25\% of SRMs are benign and even when malignant, growth rates are only approximately $0.12 \mathrm{~cm} /$ year on average. ${ }^{16,17}$

With the increased use of RFA for SRMs and lack of long-term evaluation of oncologic outcomes, consensus for follow-up, imaging surveillance, and chance of recurrence needs to be determined. In contemporary literature, local recurrence rates post-RFA have been reported from $0-19 \%{ }^{10,12,13,18-28}$

Here, we performed a retrospective review of SRMs treated with radiofrequency ablation between October 2011 and May 2019 at our academic centre. The objective of this study was to assess and review the overall rate and time to recurrence of RFA treated SRMs to better guide 
our management and follow-up strategies. Secondary objectives were to identify predicting factors selected a priori for recurrence and incomplete ablations based on expert opinion and a previous pilot study.

\section{Methods}

\section{Patient selection and predicting factors}

Institutional review board approval was obtained prior to retrospective chart review of electronic medical records of patients with a new diagnosis of a SRM who underwent radiofrequency ablation. A total of 174 recorded ablation procedures were reviewed between October 2011 and May 2019. Patients with familial syndromes at risk for multiple RCC lesions, distant metastases, biopsy proven oncocytomas, and those with less than 12 months follow-up were excluded from the dataset. Repeat RFAs of the ipsilateral kidney for incomplete ablation were not included as new ablation procedures in the context of predicting factors for tumour recurrence.

Tumours were imaged prior to RFA using either contrast-enhanced computed tomography (CT) or magnetic resonance imaging (MRI). Biopsies were available in 174 patients and done simultaneously at the time of RFA. Benign lesions were excluded from the analysis. The final cohort consisted of 141 patients. Prognostic variables were defined beforehand based on expert opinion and a previous pilot study which included patient age and gender as well as tumour size, pathology, RENAL nephrometry score and PADUA scores. RENAL nephrometry score and PADUA score was determined by two separate non-radiologist observers based on most recent imaging prior to RFA as previously defined. ${ }^{29,30}$ In the pilot study we looked at both total score and each individual component, but found no significance. Pilot study data is included in Supplementary Tables 1, 2, 3. Tumours were staged according to the American Joint Committee on Cancer TNM system.

\section{Radiofrequency ablation procedure}

All patients were seen by a urologist and referred for outpatient consultation with an interventional radiologist prior to the ablation for assessment. The parameters for the RFA procedures including probe size, energy, and roll off time were as per radiology protocols based upon original imaging and tumour size.

The LeVeen RF3000 radio-frequency ablation system (Boston Scientific) and accompanying electrodes were used in all cases. Probes were placed under CT, ultrasound or cone-beam CT guidance. Cone beam CT was utilized instead of conventional CT due to limited availability of CT time. Ablation was carried out per the IFU for renal mass ablation. Choice of probe size and use of overlapping ablations was at the operator's discretion. 


\section{Post-procedure followup}

All patients were followed with contrast-enhanced CT, MRI, or ultrasound post-RFA at intervals of 3, 6, 9, and 12 months then annually thereafter to evaluate evidence of recurrence locally and for any metastatic spread, unless poor renal function prohibited the use of contrast as determined by radiology. In general, abdominal MRI was done with or without gadolinium or CT was performed according to standard protocol with $5 \mathrm{~mm}$ sections both with and without contrast. Recurrence was defined as any sign of new or residual tumour at any point during follow-up with imaging on either CT or MRI anywhere in the originally ablated kidney. We had also predetermined to analyze a sub-group for incomplete RFA procedures which we defined as any evidence of residual disease at the ablation site on first imaging post-RFA which was to be done 3 months after the initial procedure with a CT scan as this was likely incomplete ablation rather than true recurrence. Though we attempted to standardize intervals for follow-up imaging, due to patient factors the primary measure for duration to recurrence was measured in months rather than number of negative imaging studies. This sub-group selection was based on our pilot study which showed that larger tumours tended to have a higher frequency of recurrence, but that they were all in the first 3-6 months and did not have a disease-free period before recurrence. Though complications were not recorded in a standardized fashion at the time of procedure, each chart was reviewed for any significant complications post-procedure based on follow-up clinical visits.

\section{Statistical analysis}

Categorical variables are reported here through descriptive statistics including frequencies and proportions. Means and medians with standard deviation and interquartile ranges are presented for continuous variables.

Univariate and multivariate Cox proportional hazard analyses were completed to assess for predicting factors between patient factors (age, gender) and tumour characteristics (tumour size, location, biopsy pathology) with recurrence and incomplete ablations. Tumour size was analyzed in increments of $0.1 \mathrm{~cm}$ corresponding to the degree of specificity of the size reported on conventional imaging. For the multivariate analysis included all significant variables and one variable to include with the smallest $p$-value in addition to tumour size to evaluate if the results remained significant and independent in all groups and sub-group analysis. We initially included the RENAL and PADUA scores in our pilot study as predictive factors, but the data did not show any significant predictive value with respect to tumour recurrence (Supplementary Tables 1, 2, 3). Sub-group analysis was completed for patients with new tumour recurrences as well as for those with residual tumour following first RFA procedure as described above.

All statistical analyses were performed using IBM $^{\circledR}$ SPSS $^{\circledR}$ Statistics software package (IBM Corp. Released 2015. IBM SPSS Statistics for Windows, Version 23.0. Armonk, NY: IBM Corp). 


\section{Results}

Baseline patient demographic and tumour characteristics are reported in Table 1. Most patients who had biopsy before the procedure had pathology in agreement with clear cell renal cell carcinoma (51.1\%). Of note, 44 patients either had biopsies which were non-diagnostic or not done before RFA and we chose to include these patients in the study. Though 22 patients were shown to have papillary RCC, their charts did not reflect whether these were specially type I or type II. Overall average tumour size was $2.57 \pm 0.84 \mathrm{~cm}$. Sub-group analysis showed that although there was evidence that patients who had residual disease or incomplete ablation had higher average tumour size than those in the new recurrence group, this did not reach statistical significance when comparing the two groups $(3.53 \pm 1.22 \mathrm{~cm}$ vs. $2.69 \pm 0.75 \mathrm{~cm} ; \mathrm{p}=0.08)$. Table 3 shows the distribution of recurrences based on tumour size.

Thirty-three patients had benign lesions (32 oncocytomas and one angiomyolipoma and were not included in the 141 patients reviewed. Median time to recurrence was 15 months (range 6 - 53 months) with no recurrences occurring beyond 53 months post-RFA. Of the 141 patients, $9(6.4 \%)$ had new recurrences and $13(9.2 \%)$ had evidence of residual disease or incomplete ablation during the follow-up period. In total 126 patients $(89.4 \%)$ were initially evaluated with CT scans and the remaining $15(10.6 \%)$ were initially evaluated with MRI. Median length of follow-up for all patients was 67 months (range: $18-161$ ). Three of the 9 new recurrences $(33.3 \%)$ had successful repeat RFA procedures, 4 (44.4\%) were kept on AS, one had a radical nephrectomy, and one patient progressed to metastatic disease after surveillance and chose to have no further treatment. Six of the 13 patients $(46.1 \%)$ with residual disease had successful repeat RFA, while $3(18.8 \%)$ went on to radical nephrectomy, two $(15.4 \%)$ elected for AS, one $(7.7 \%)$ was treated with sunitinib for metastatic disease likely due to pT3 disease in the contralateral kidney previously resected, and one patient was lost to follow-up. Median follow-up of all patients was 67 (range 18 -161) months.

On univariate and multivariate analysis (Table 2 ), tumour size was positively associated with recurrence disease $(\mathrm{HR}=2.158 ; 95 \% \mathrm{CI}=1.477-3.153 ; \mathrm{p}<0.001)$. Sub-group analysis for either incomplete ablation or residual disease (Table 4) was also positively associated with tumour size $(\mathrm{HR}=2.256 ; 95 \% \mathrm{CI}=1.461-3.512 ; p<0.001)$, but this was not shown for new recurrence $($ Table 5$)(\mathrm{HR}=1.395 ; 95 \% \mathrm{CI}=0.629-3.095 ; \mathrm{p}=0.436)$.

No clinically significant complications ( $\geq$ Clavian-Dindo III) were reported which required subsequent intervention.

\section{Discussion}

SRMs are increasing in incidence likely due to the increasing accessibility and reduction in cost of axial imaging results in more incidental findings. The current gold standard treatment for renal cell carcinoma has been by either partial or radical nephrectomy, but radiofrequency ablation is being used more commonly over the last twenty years for SRMs. ${ }^{2,8,10-13}$ RFA is a less invasive 
procedure that can be beneficial for patients who are poor surgical candidates, have declined AS, or in which AS is inappropriate. No clinically significant complications were reported based on our data; however, we acknowledge that this is limited by the retrospective nature of the review. Shared decision-making should be undertaken with patients to discuss the potential benefits and risks of RFA versus other modalities of treatment or surveillance. These include RFAs less invasive nature, but lack of long-term data and potential need for secondary intervention. While there are various guidelines on follow-up and imaging post-partial or radical nephrectomy for RCC there have been few on RFA specific follow-up.

Our original pilot study was designed to gather information on recurrences rates in our patients who underwent RFA and determine if tumour characteristics could be used as a predictor for patients who are at risk for recurrence. At first, we attempted to use more complex scoring systems such as the RENAL nephrometry and PADUA scores, but they did not show any significant benefit for prediction of tumour recurrence or residual tumour post-RFA over maximum tumour size alone. Here we completed a larger review of our patients with long-term follow-up after RFA and again showed that larger tumours appear to be a positive predictor of recurrence with a hazard ratio of 2.256. Patients who had recurrences had statistically significantly larger tumours $(3.53 \pm 1.22 \mathrm{~cm}$ vs. $2.47 \pm 0.72 \mathrm{~cm})$. This is explained by subgroup analysis for patients who had incomplete ablation post-RFA which showed significance of tumour size and not seen in patients with true new recurrences during their follow-up. This suggests that though tumour size may be helpful in determining which patients may require a secondary procedure after RFA, it is still unclear whether this can be used to determine patients at higher risk for recurrence following successful treatment.

The original design and purpose of this study was to identify patients who had recurrence of RCC post-RFA therapy to help guide follow-up strategies overall our recurrence rates $(15.6 \%)$ were within what is reported in contemporary literature for partial and radical nephrectomy. It is important to highlight from this data that most of these recurrences were a result of incomplete ablation (13/22 cases). Only 1 patient with residual disease went on to eventual progression of metastatic disease and the rest had secondary procedures with either a repeat RFA, nephrectomy, or continued with AS.

Of all recurrences, the median time to recurrence was 15 months with no recurrences occurred after 53 months. This suggests that most recurrence are likely to occur within the first few years after RFA and that follow-up for RCC post-RFA can be similar to that of RCC postsurgical resection after 1-2 years. Post RFA surveillance can possibly be discontinued after 5 years, but further follow-up is needed for confirmation.

The authors acknowledge that there are limitations to this study which include the retrospective nature, single centre patient population, lack of pathologic confirmation of RCC in 44/141 (31\%) of patients, and smaller sample size for regression analysis. As a result, we cannot comment on a 
cut-off tumour size that would significantly increase risk of incomplete ablation or future recurrence, but with larger studies and more data this could be investigated in the future.

\section{Conclusions}

Overall, this study shows the risk of recurrence following RFA for SRMs is $15.6 \%$. Most recurrences were a result of incomplete ablation at the original site identified within the first 8 months post-RFA at $9.2 \%$ and that tumour size is predictive of this occurrence. Tumour size alone, without need for complex scoring systems, may serve as a predictor of incomplete ablation following RFA and could be used to assist in shared decision making on management strategies. No recurrences were identified beyond 53 months with a total median follow-up time of 67 months and a modified follow-up imaging schedule post-surgical resection for nonmetastatic RCC can be used with more frequent imaging in the first year. This report suggests that post RFA surveillance may be discontinued after 5 years, but further follow-up is needed to confirm this. 


\section{References}

1. Gill IS, Aron M, Gervais DA, Jewett MAS. Clinical practice. Small renal mass. N Engl J Med. 2010;362(7):624-634. doi:10.1056/NEJMcp0910041

2. Ljungberg B, Bensalah K, Bex A. Guidelines on Renal Cell Carcinoma. Guidelines on Renal Cell Carcinoma. http://uroweb.org/guideline/renal-cell-carcinoma/. Published 2018. Accessed May 20, 2018.

3. Campbell S, Uzzo RG, Allaf ME, et al. Renal Mass and Localized Renal Cancer: AUA Guideline. J Urol. 2017;198(3):520-529. doi: 10.1016/j.juro.2017.04.100

4. Canadian Cancer Society Canadian Cancer Statistics. 2017. http://www.cancer.ca/ /media/cancer.ca/CW/cancer\%20information/cancer\%20101/Ca nadian $\% 20$ cancer\%20statistics/Canadian-Cancer-Statistics-2017-EN.pdf?la=en. Accessed May 20, 2018.

5. Hollingsworth JM, Miller DC, Daignault S, Hollenbeck BK. Rising incidence of small renal masses: a need to reassess treatment effect. J Natl Cancer Inst. 2006;98(18):13311334. doi:10.1093/jnci/djj362

6. Drangsholt S, Huang WC. Current Trends in Renal Surgery and Observation for Small Renal Masses. Urol Clin North Am. 2017;44(2):169-178. doi: 10.1016/j.ucl.2016.12.003

7. Kassouf W, Monteiro LL, Drachenberg DE, et al. Canadian Urological Association guideline for followup of patients after treatment of non-metastatic renal cell carcinoma. Can Urol Assoc J. 2018;12(8):231-238. doi:10.5489/cuaj.5462

8. Jewett MAS, Rendon R, Lacombe L, et al. Canadian guidelines for the management of small renal masses (SRM). Can Urol Assoc J. 2015;9(5-6):160-163. doi:10.5489/cuaj.2969

9. McGovern FJ, Wood BJ, Goldberg SN, Mueller PR. Radio frequency ablation of renal cell carcinoma via image guided needle electrodes. J Urol. 1999;161(2):599-600.

10. Park S, Anderson JK, Matsumoto ED, Lotan Y, Josephs S, Cadeddu JA. Radiofrequency ablation of renal tumors: intermediate-term results. J Endourol. 2006; 20:569-573. doi:10.1089/end.2006.20.569

11. D'Andrea D, Shariat SF, Klatte T. Update on ablative therapies of renal tumors. Curr Opin Urol. 2016; 26:410-416. doi:10.1097/MOU.0000000000000306

12. Psutka SP, Feldman AS, McDougal WS, McGovern FJ, Mueller P, Gervais DA. Longterm oncologic outcomes after radiofrequency ablation for T1 renal cell carcinoma. Eur Urol. 2013; 63:486-492. doi: 10.1016/j.eururo.2012.08.062

13. Campbell SC, Novick AC, Belldegrun A, et al. Guideline for management of the clinical T1 renal mass. J Urol. 2009;182(4):1271-1279. doi: 10.1016/j.juro.2009.07.004

14. Sun M, Bianchi M, Trinh Q-D, et al. Comparison of partial vs radical nephrectomy with regard to other-cause mortality in T1 renal cell carcinoma among patients aged $\geq 75$ years with multiple comorbidities. BJU Int. 2013;111(1):67-73. doi:10.1111/j.1464410X.2012. 11254.x

15. Johnson B, Sorokin I, Cadeddu JA. Ten-year outcomes of renal tumor radiofrequency ablation. J Urol. August 2018. doi: 10.1016/j.juro.2018.08.045 
16. Leveridge MJ, Finelli A, Kachura JR, et al. Outcomes of small renal mass needle core biopsy, nondiagnostic percutaneous biopsy, and the role of repeat biopsy. Eur Urol. 2011;60(3):578-584. doi: 10.1016/j.eururo.2011.06.021

17. Organ M, Jewett M, Basiuk J, et al. Growth kinetics of small renal masses: A prospective analysis from the Renal Cell Carcinoma Consortium of Canada. Can Urol Assoc J. 2014;8(1-2):24-27. doi:10.5489/cuaj.1483

18. Zagoria RJ, Traver MA, Werle DM, Perini M, Hayasaka S, Clark PE. Oncologic efficacy of CT-guided percutaneous radiofrequency ablation of renal cell carcinomas. AJR Am J Roentgenol. 2007; 189:429-436. doi:10.2214/AJR.07.2258

19. Georgiades CS, Rodriguez R. Efficacy and safety of percutaneous cryoablation for stage 1A/B renal cell carcinoma: results of a prospective, single-arm, 5-year study. Cardiovasc Intervent Radiol. 2014;37(6):1494-1499. doi:10.1007/s00270-013-0831-8

20. Ma Y, Bedir S, Cadeddu JA, Gahan JC. Long-term outcomes in healthy adults after radiofrequency ablation of T1a renal tumours. BJU Int. 2014;113(1):51-55. doi:10.1111/bju.12366

21. Wah TM, Irving HC, Gregory W, Cartledge J, Joyce AD, Selby PJ. Radiofrequency ablation (RFA) of renal cell carcinoma (RCC): experience in 200 tumours. BJU Int. 2014;113(3):416-428. doi:10.1111/bju.12349

22. Ramirez D, Ma Y-B, Bedir S, Antonelli JA, Cadeddu JA, Gahan JC. Laparoscopic radiofrequency ablation of small renal tumors: long-term oncologic outcomes. $J$ Endourol. 2014;28(3):330-334. doi:10.1089/end.2013.0542

23. Best SL, Park SK, Youssef RF, et al. Long-term outcomes of renal tumor radio frequency ablation stratified by tumor diameter: size matters. $J$ Urol. 2012;187(4):11831189. doi: 10.1016/j.juro.2011.11.096

24. Olweny EO, Park SK, Tan YK, Best SL, Trimmer C, Cadeddu JA. Radiofrequency ablation versus partial nephrectomy in patients with solitary clinical T1a renal cell carcinoma: comparable oncologic outcomes at a minimum of 5 years of follow-up. Eur Urol. 2012;61(6):1156-1161. doi: 10.1016/j.eururo.2012.01.001

25. Takaki H, Soga N, Kanda H, et al. Radiofrequency ablation versus radical nephrectomy: clinical outcomes for stage T1b renal cell carcinoma. Radiology. 2014;270(1):292-299. doi:10.1148/radiol.13130221

26. Atwell TD, Schmit GD, Boorjian SA, et al. Percutaneous ablation of renal masses measuring $3.0 \mathrm{~cm}$ and smaller: comparative local control and complications after radiofrequency ablation and cryoablation. AJR Am J Roentgenol. 2013;200(2):461-466. doi:10.2214/AJR. 12.8618

27. Chen Y, Huang J, Xia L, et al. Monitoring laparoscopic radiofrequency renal lesions in real time using contrast-enhanced ultrasonography: an open-label, randomized, comparative pilot trial. J Endourol. 2013;27(6):697-704. doi:10.1089/end.2012.0694

28. Schmit GD, Thompson RH, Kurup AN, et al. Usefulness of R.E.N.A.L. nephrometry scoring system for predicting outcomes and complications of percutaneous ablation of 751 renal tumors. J Urol. 2013;189(1):30-35. doi: 10.1016/j.juro.2012.08.180 
29. Kutikov A, Uzzo RG. The R.E.N.A.L. nephrometry score: a comprehensive standardized system for quantitating renal tumor size, location and depth. J Urol. 2009; 182:844-853. doi: 10.1016/j.juro.2009.05.035

30. Ficarra V, Novara G, Secco S, et al. Preoperative aspects and dimensions used for an anatomical (PADUA) classification of renal tumours in patients who are candidates for nephron-sparing surgery. Eur Urol. 2009; 56:786-793. doi:

10.1016/j.eururo.2009.07.040

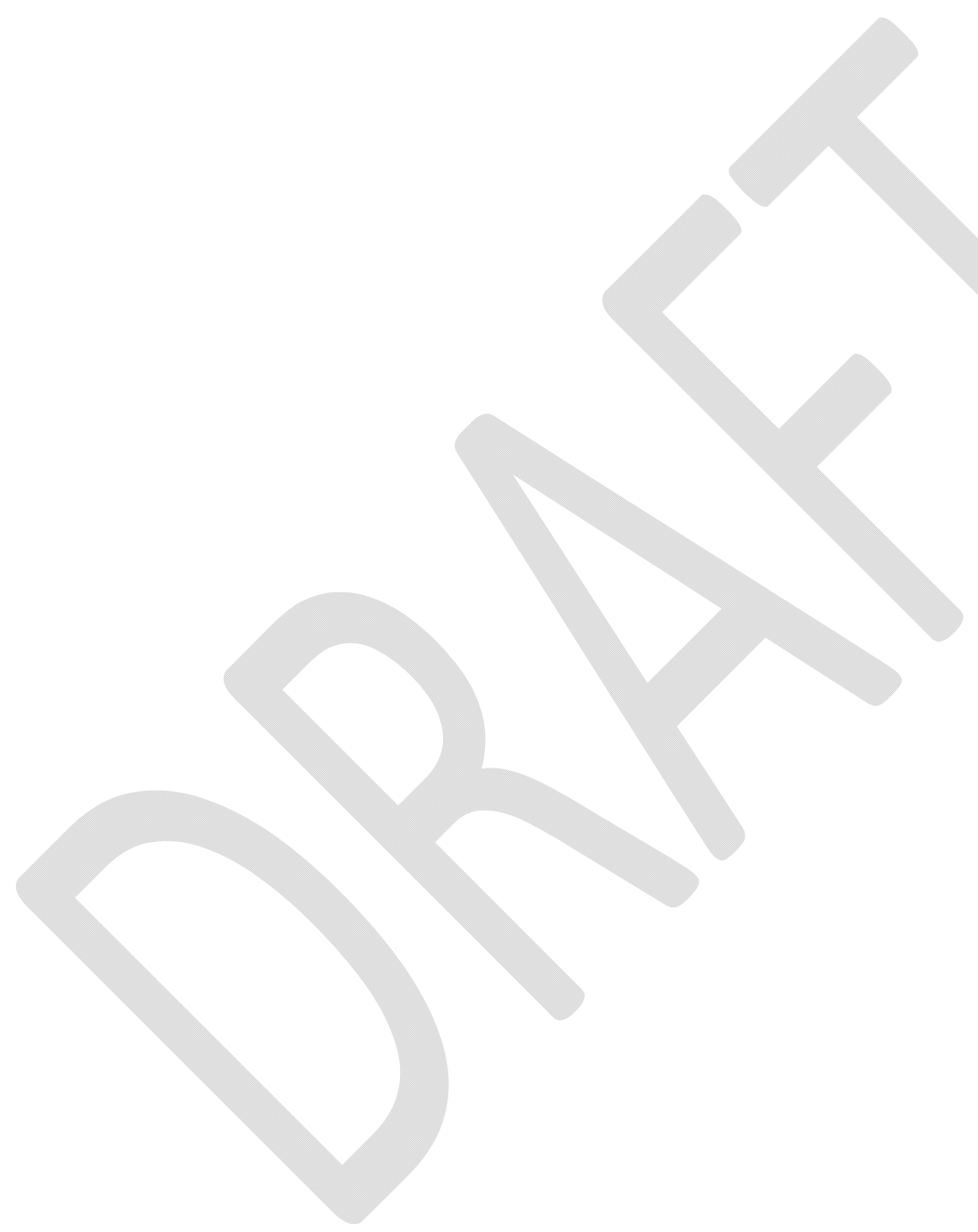


Figures and Tables

\begin{tabular}{|l|c|}
\hline Table 1. Patient demographics \\
\hline Parameter & Variable \\
\hline Cases & 141 \\
\hline Mean age & $69.0 \pm 11.1$ \\
\hline \# new recurrences & $9(6.4 \% \%)$ \\
\hline \# incomplete ablations & $13(9.2 \%)$ \\
\hline \# total recurrences & $22(15.6 \%)$ \\
\hline Median followup (range) & $67.0(18.0-161.0)$ months \\
\hline Gender & 101 male $(71.6 \%), 40$ female $(28.4 \%)$ \\
\hline Mean tumor size & $2.57 \pm 0.84 \mathrm{~cm}$ \\
\hline Pathology & $\begin{array}{c}72 \text { clear-cell RCC }(51.1 \%), 44 \text { non- } \\
\text { diagnostic }(31.2 \%), 22 \text { papillary RCC } \\
(15.6 \%), 3 \text { chromophobe }(2.1 \%)\end{array}$ \\
\hline
\end{tabular}

RCC: renal cell carcinoma.

\begin{tabular}{|c|c|c|c|c|c|c|}
\hline \multirow[b]{2}{*}{ Parameter } & \multicolumn{3}{|c|}{ Univariate analysis } & \multicolumn{3}{|c|}{ Multivariate analysis } \\
\hline & HR & $95 \% \mathrm{CI}$ & $\mathbf{p}$ & HR & $95 \% \mathrm{CI}$ & p \\
\hline Age & 1.017 & $0.977-1.058$ & 0.408 & 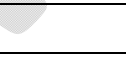 & & \\
\hline Gender & 0.925 & $0.362-2.364$ & 0.871 & & & \\
\hline Tumor size & 2.158 & $1.477-3.153$ & $<0.001$ & 2.027 & 1.369-3.001 & $<0.001$ \\
\hline Tumor side & 1.917 & $0.781-4.703$ & 0.155 & 1.005 & $0.971-1.041$ & 0.771 \\
\hline
\end{tabular}

CI: confidence interval; HR: hazard ratio. 


\begin{tabular}{|l|c|c|c|}
\hline Table 3. Distribution of recurrence by tumor size \\
\hline Tumour size & \multicolumn{3}{|c|}{ N } \\
\hline & $\begin{array}{c}\text { True } \\
\text { recurrence }\end{array}$ & $\begin{array}{c}\text { Incomplete } \\
\text { ablation }\end{array}$ & $\begin{array}{c}\text { No } \\
\text { recurrence }\end{array}$ \\
\hline$<1 \mathrm{~cm}$ & 0 & 0 & 0 \\
\hline $1-2 \mathrm{~cm}$ & 2 & 0 & 37 \\
\hline $2-3 \mathrm{~cm}$ & 4 & 8 & 62 \\
\hline$>3 \mathrm{~cm}$ & 3 & 5 & 20 \\
\hline
\end{tabular}

\begin{tabular}{l} 
Table 4. Cox proportional hazard regression analysis for incomplete ablation following \\
RFA \\
\hline
\end{tabular}

CI: confidence interval; HR: hazard ratio; RFA: radiofrequency ablation.

\begin{tabular}{|c|c|c|c|}
\hline & 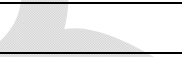 & Univariate anal & \\
\hline Parameter & HR & $95 \% \mathrm{CI}$ & $\mathbf{p}$ \\
\hline Age & 1.000 & $0.942-1.062$ & 0.998 \\
\hline Gender & 0.695 & $0.144-3.348$ & 0.650 \\
\hline Tumor size & 1.395 & $0.629-3.095$ & 0.413 \\
\hline Tumor side & 1.872 & $0.468-7.489$ & 0.375 \\
\hline
\end{tabular}

CI: confidence interval; HR: hazard ratio; RFA: radiofrequency ablation. 
Appendix A. Pilot study data

\begin{tabular}{|l|c|}
\hline \multicolumn{2}{|l|}{ Supplementary Table 6. Patient demographics } \\
\hline Parameters & Variables \\
\hline Cases (n) & 84 \\
\hline Mean age & 59 male, 25 female \\
\hline Gender & $2.42 \pm 0.81 \mathrm{~cm}$ \\
\hline Tumor size & $\begin{array}{c}40 \text { clear cell, } 16 \text { papillary } \mathrm{RCC}, 3 \\
\text { chromophobe, } 25 \text { not completed }\end{array}$ \\
\hline Pathology & $6.81 \pm 1.58$ \\
\hline RENAL nephrometry score & $8.13 \pm 1.39$ \\
\hline PADUA score &
\end{tabular}

RCC: renal cell carcinoma.

\begin{tabular}{|l|c|c|c|c|c|c|}
\hline $\begin{array}{l}\text { Supplementary Table 7. Cox proportional hazard regression analysis for residual } \\
\text { tumor following RFA }\end{array}$ & \multicolumn{5}{|c|}{ Univariate analysis } & \multicolumn{3}{c|}{ Multivariate analysis } \\
\hline & HR & $\mathbf{9 5 \%}$ CI & p & HR & $\mathbf{9 5 \%}$ CI & p \\
\hline Parameters & 1.03 & $0.94-1.13$ & 0.582 & & & \\
\hline Mean age & 1.61 & $0.27-9.60$ & 0.604 & & & \\
\hline Gender & $\mathbf{2 . 4 0}$ & $\mathbf{1 . 0 1 - 5 . 7 1}$ & $\mathbf{0 . 0 4 7}$ & 2.13 & $0.81-5.63$ & 0.127 \\
\hline Mean tumor size & 2.95 & $0.53-16.41$ & 0.217 & 1.05 & $0.08-13.45$ & 0.973 \\
\hline RENAL score & 2.62 & $0.64-10.78$ & 0.183 & 1.98 & $0.26-15.33$ & 0.512 \\
\hline PADUA score & &
\end{tabular}

CI: confidence interval; HR: hazard ratio; RFA: radiofrequency ablation. 


\begin{tabular}{|l|l|c|c|}
\hline $\begin{array}{l}\text { Supplementary Table 8. Cox proportional hazard regression analysis for new } \\
\text { tumor recurrence following RFA }\end{array}$ & \multicolumn{3}{|c|}{ Univariate analysis } \\
\hline & HR & $\mathbf{9 5 \%}$ C & p \\
\hline Parameters & 1.01 & $0.92-1.10$ & 0.900 \\
\hline Mean age & 0.03 & $0-354.34$ & 0.464 \\
\hline Gender & 1.27 & $0.41-3.93$ & 0.684 \\
\hline Mean tumor size & 1.21 & $0.21-6.95$ & 0.830 \\
\hline RENAL score & 1.56 & $0.38-6.43$ & 0.541 \\
\hline PADUA score & \multicolumn{3}{|c|}{} \\
\hline
\end{tabular}

CI: confidence interval; HR: hazard ratio; RFA: radiofrequency ablation. 